\title{
Characterization of the Martian Convective Boundary Layer
}

\author{
Germán Martínez * \\ Universidad Complutense de Madrid, Madrid, Spain \\ Francisco VALERo \\ Universidad Complutense de Madrid, Madrid, Spain \\ LuIS VÁzQUEz \\ Universidad Complutense de Madrid, Madrid, Spain
}

Journal of Atmospheric Sciences, in press.

\begin{abstract}
The authors have carried out an extensive characterization of the Martian Mixed Layer formed under convective conditions. The values of the mixed layer height, convective velocity scale, convective temperature scale, mean temperature standard deviation, mean horizontal and vertical velocity standard deviations, and mean turbulent viscous dissipation rate have been obtained during the strongest convective hours for the mixed layer. In addition, the existing database of the surface layer has been improved by recalculating some parameters -such as Monin-Obukhov length, friction velocity, or scale temperature- which had already been obtained in previous papers by other means, and also by calculating new ones, such as vertical velocity wind speed standard deviation, turbulent viscous dissipation rate, and eddy transfer coefficients for momentum and heat. Counterpart Earth values of all these magnitudes are also shown. All together, throughout the paper, a comprehensive database concerning the whole Convective Planetary Boundary Layer on Mars is displayed, and also a detailed terrestrial comparison is established.
\end{abstract}

\footnotetext{
*Corresponding author address: Germán Martínez, Dept. de Matemática Aplicada, Facultad de Informtica, Universidad Complutense de Madrid, Av. Complutense s/n, Madrid, MA 28040, Spain.

E-mail: mmgerman@fis.ucm.es
}

The inputs of this work are hourly in situ temperature, hourly in situ horizontal wind speed, and hourly simulated ground temperature for specific selected Sols of Vikings and Pathfinder landers. These data correspond to typical low and mid latitude northern summer time conditions, with weak prevailing winds. To handle this set of data, surface layer and mixed layer similarity theory have been used at the strongest convective hours. In addition, the inclusion of a parametrization of a molecular sublayer, and prescribed values of the surface roughness have been considered.

\section{Introduction}

The mixed layer is one of the three layers into which the convective boundary layer can be divided, with the surface layer and the entrainment zone lying beneath and above it, accordingly. It is characterized by an intense vertical mixing which tends to leave variables such as potential temperature and humidity nearly constant with height, even wind speed and direction 31. On Earth, it typically encompasses between 40-70 \% of the convective boundary layer height. The Martian planetary boundary layer (MPBL), and specifically the Martian mixed layer (MML), is one of the least known parts of the Martian atmosphere. This is so, among other minor aspects, due to the lack of in situ data covering the MML, to some specific Martian features like the atmospheric dust load, and to its own intrinsic turbulent nature. Nevertheless, its understanding be- 
comes quite important for several reasons. It is the place through which current and future robotic and manned missions will be sent. Therefore, at least partial knowledge of its dynamical and thermal behavior is needed for the design of both the spacecrafts and sensors. In addition, small scale processes "belonging" to the MML have an impact on larger scale processes, being the reverse also true. As a consequence, General Circulation Models and mesoscale models need to incorporate MML effects to simulate more accurately the real atmospheric behavior.

Up to now, there are three kinds of papers referring to the MPBL literature. The first one concerns analytical 1D models (8], 3], 15], [37, 22, [9], 23], 24], 25], and [14]), 2D models (26], 28], and 18 ) and 3D models (21, and 35). These papers (1D, 2D, and 3D models) show three kinds of results: (i) vertical profiles of temperature, wind speed, heating rates (radiative and turbulent), and kinematic heat flux at specific hours, (ii) diurnal evolutions of magnitudes that have been measured in situ to test the model, that is, temperature and wind speed near the ground, and (iii) the energy balance at the ground. However, no MML turbulent parameters (turbulent statistics, fundamental mixed layer similarity scales, dissipation rate across the MML...) are shown through them.

The second kind of papers refers to surface layer similarity models, which need simulated ground temperature and in situ wind and temperature measurements at the first meters (32, 34, 12, and [16]). They all use surface layer similarity theory, and, therefore, only Martian surface layer (MSL) parameters are calculated, except for the mixed layer height.

Large Eddy Simulation models (LES's) form the third kind of papers (21], 17, 36], 29], and [30). In principle they are supposed to perform with the highest accuracy throughout the MML, although they undertake an expensive computational time. Taking a detailed insight into them, 21] shows vertical wind speed variance $\sigma_{w}^{2}(z, t)$ and turbulent kinetic energy $e(z, t)$ as a function of height and time, albeit for a generic run [see page 246 of 21] for the details of the generic run]. [17, for Pathfinder conditions, comment on the qualitative behavior of $\sigma_{w}^{2}(z, t)$ and $e(z, t)$, but none of the MML magnitudes which we present here are displayed. On the other hand, 29] and 30] do show turbulent statistics and fundamental mixed layer similarity scales (mixed layer height, convective velocity scale, and convective temperature scale), although for a generic run (see Table 2 of that paper) and in a dimensionless form.

Noticing the current state-of-the-art, we have carried out a specific research of the MML by calculating the characteristic parameters of the mixed layer: mixed layer height $z_{i}$, convective velocity scale $w_{*}$, convective temperature scale $\theta_{*}$, mean horizontal $\left\langle\sigma_{u}\right\rangle$ and vertical $\left\langle\sigma_{w}\right\rangle$ velocity standard deviations, mean temperature standard deviation $\left\langle\sigma_{\theta}\right\rangle$, and mean turbulent viscous dissipation rate $\langle\epsilon\rangle$, where the bars denote averaging over the whole mixed layer. Our second goal has been to review and complete the existing Martian Surface Layer (MSL) database. In reviewing it, we have recalculated some MSL parameters, namely: Monin Obukhov length $L$, friction velocity $u_{*}$, and temperature scale $T_{*}$. These magnitudes had already been estimated by other means in previous papers, see [16] for a complete MSL review. In completing the MSL database, we have derived -for the first time from in situ data and similarity relationshipsnew MSL magnitudes, such as turbulent viscous dissipation rate $\epsilon^{z}$, standard deviation of vertical wind speed $\sigma_{w}^{z}$, and eddy transfer coefficients for heat $k_{h}^{z}$, and momentum $k_{m}^{z}$, where the superscript $\mathrm{z}$ denotes the height at which they have been calculated $(1.3 \mathrm{~m}$ for the Pathfinder, and $1.6 \mathrm{~m}$ for the Viking landers). In short, this paper displays an extensive database covering the whole MPBL, from the MSL up to the MML. In addition, references and typical values of all the counterpart terrestrial magnitudes are given to allow a direct comparison with the terrestrial boundary layer. Definitions and conceptual meaning of each of the magnitudes calculated above can be seen in Appendix A.

Even at present, Vikings and Pathfinder missions still provide the most suitable in situ data to perform micrometeorological research. Static simultaneous measurements of wind speed and atmospheric temperature monitored with a high sample, and during times of the order of 1 hour without interruptions, have just been taken by these two missions. Mars Exploration Rover and Phoenix data are also useful, although they do not satisfy the restrictions above. This is why we have calculated all these magnitudes at Vikings (VK's) and Pathfinder (PF) sites. To do it, we have used: 
(i) in situ temperature and horizontal wind speed data, (ii) simulated ground temperature, and (iii) surface layer and mixed layer similarity theory together with a parametrized molecular sublayer and prescribed values of the surface roughness.

A warning about the nature of these data must be stated at this point. As it will be commented in the next section, the observations of this work belong to typical low mid latitude northern summer time Sols. Under such conditions, mean wind is very weak, and consequently the mixed layer formed is mainly convectively driven. This was the case at VK's and PF selected Sols, given the small influence of the atmospheric distortion and variability that took place there. Therefore, our results are generic for such conditions.

Section 2 deals with the data that form the inputs of this work. There we explain why they are the most accurate ones representing VK's and PF sites. In section 3 , we analyze the methodology used to derive the results while the results of this paper are presented in section 4. This, in turn, is divided into three subsections, namely: the fundamental similarity scales, the turbulent statistics, and turbulent viscous dissipation rate together with eddy diffusivity coefficients for heat and momentum. In each of them, the results are shown both for the MML and for the MSL. Section 5 splits also into three subsections. In the first one, we give a comparison between the MML and MSL values. A description of the sensitivity of the results to the inclusion of a molecular sublayer, to the surface roughness, and finally to the created ground temperature scenarios is shown and explained in next subsection. The third subsection that completes section 5 shows counterpart terrestrial values of all our derived magnitudes, and there is also a comment about the existing analogies and differences between the two planets. The paper ends with section 6 , where we offer a brief summary of the paper, and we propose the main conclusions.

\section{Data}

Three sets of data form the inputs of this paper: in situ temperature and in situ horizontal wind speed (at $1.3 \mathrm{~m}$ for the PF and $1.6 \mathrm{~m}$ for the VK's), together with hourly simulated ground temperature. They all belong to some selected Sols of VK's and
PF missions. To get a detailed information on both missions, see 10] and 27.

As the reliability of this work strongly depends on the quality of the inputs, we will detail why and how we have selected them. Sols 27, 28, and 35 of Viking Lander 1 (VL1), Sols 20 and 25 of Viking Lander 2 (VL2), and Sol 25 of PF were chosen. They correspond to a solar longitude of about $110^{\circ}, 127^{\circ}$, and $155^{\circ}$ for the VL1, VL2, and PF Sols, respectively. In taking VL's Sols, the following were taken into account: lander interferences, sensors sampling rate, the accuracy of the sensors, the time of the year, and the amount of atmospheric dust. Data belonging to the above VK's Sols fulfill the most optimal conditions. That is, prevailing wind direction involved the least lander interferences. On the other hand, wind speed and temperature sampling rate during the strongest convective hours was the highest possible (which implies more accurate means and variances). In addition, the accuracy of the sensors was at its highest level (see Table 11). It was northern summertime and

Table 1: Measurement errors at the Viking locations.

\begin{tabular}{|lll|ll|}
\hline \multicolumn{3}{|c|}{ Sols 1-44 } & Sols > 44 \\
\hline & $T$ & $U$ & $T$ & $U$ \\
VL-1 & $\pm 1.5 K$ & $\pm 10 \%$ & Good tempt & Degraded \\
VL-2 & $\pm 2 K$ & $\pm 10 \%$ & Problems & $\pm 10 \%$ \\
\hline
\end{tabular}

hence, baroclinic disturbances, which could cause a worse perform of the similarity theory and a not convectively driven mixed layer, were not present. Finally, the amount of atmospheric dust was at its minimum, which could also hinder the application of similarity theory. With regards to PF Sol 25, the reason for which it has been selected is easier to describe. This is the only Sol in which temperature and horizontal velocity measurements are available to us during a entire Sol. In addition, they also correspond to typical summer time conditions, which is needed in this work.

Specifically, for the VK's Sols, mean hourly values of horizontal wind speed and temperature are straightforwardly shown in NASA Planetary Data System (PDS). Also the sensors sampling rate (number of measurements each hour) are shown in 
it. On the other hand, only $0.25 \mathrm{~Hz}$ temperature measurements are found in the PDS corresponding to PF Sol 25. Horizontal wind speed measurements, also monitored at $0.25 \mathrm{~Hz}$ during the entire Sol, have been kindly provided by Dr. Jim Murphy, as the wind sensor experienced problems. Thus, we have just hourly averaged these temperature and horizontal wind speed data to obtain the hourly outputs in the PF case. As a result, in Figs. 11 and 2 we can appreciate the temperature and horizontal wind speed data corresponding to the Sols under study.

Special care has been taken when considering the ground temperature $T_{g}$, since it is the only non-insitu input. We have simulated $T_{g}$ corresponding to all the Sols under study, see Fig. [3] by using a modified version of the 1D model 25. The procedure followed to derive the ground temperature for the selected Sols has been a tricky issue. Appendix B was created to clarify how this magnitude has been obtained.

\section{Methodology}

Let us show the methodology employed to obtain our results. The way followed to derive $L, u_{*}, T_{*}$, $\epsilon^{z}, k_{m}^{z}$, and $k_{h}^{z}$ from our inputs is highly detailed in the companion paper [16. Turning to the MSL turbulent statistics, we have calculated $\sigma_{w}^{z}$ by using the surface layer similarity relationship

$$
\frac{\sigma_{w}^{z}}{u_{*}}=1.3(1+3(-z / L))^{1 / 3}
$$

given by 19 . On the other hand, horizontal velocity standard deviation, $\sigma_{u}^{z}$, and temperature standard deviation, $\sigma_{T}^{z}$, have been directly inferred from the in situ temperature and horizontal velocity measurements belonging to VK's and PF missions.

Once the MSL parameters have been obtained, the relation that enables us to link the MSL to the MML is

$$
\frac{\sigma_{u}^{z}}{u_{*}}=\left(12+0.5\left(z_{i} /|L|\right)\right)^{1 / 3}
$$

given by [19. Here it has been supposed that the horizontal velocity standard deviation scales with the convective velocity scale $w_{*}$, because the horizontal component of the convective eddies is expected to extend down to the surface. Eq. (2)
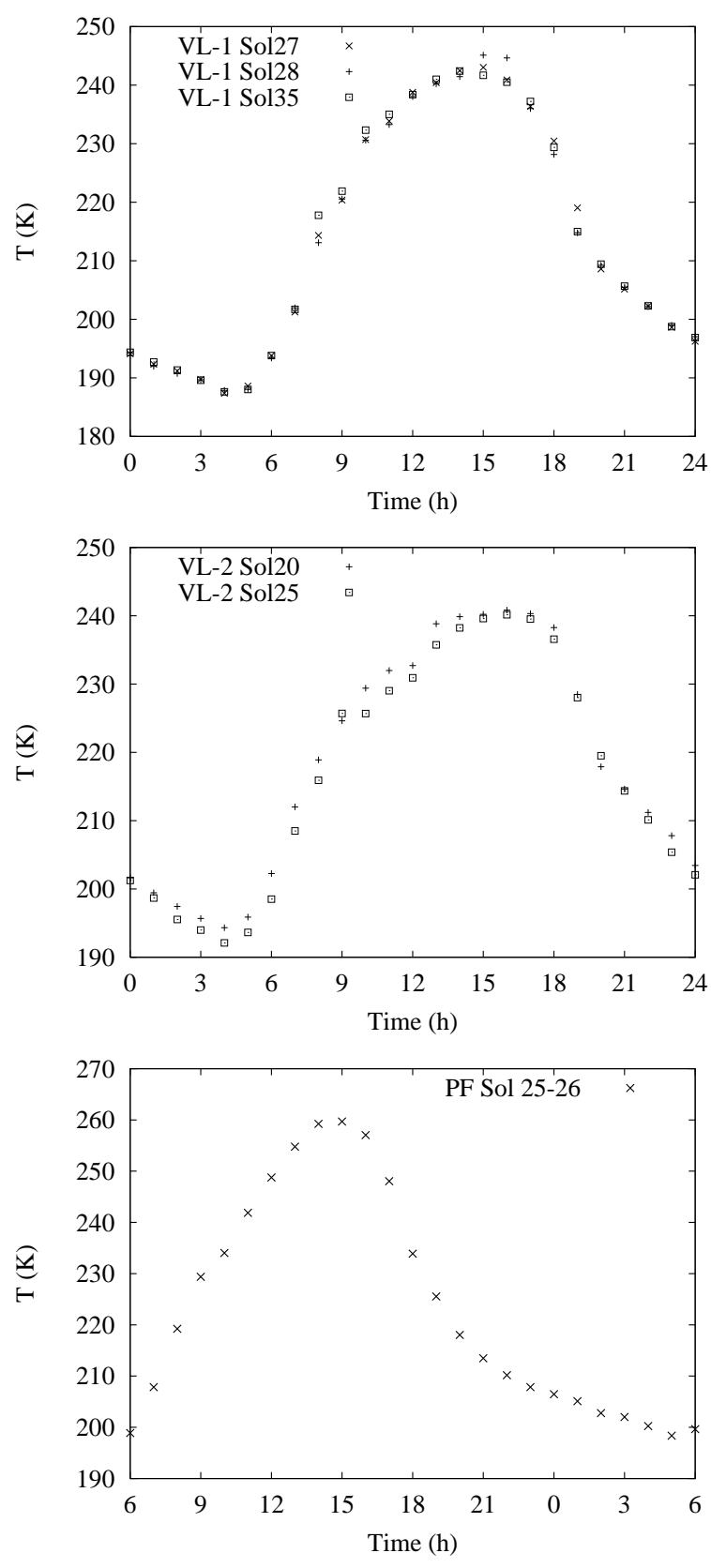

Figure 1: In situ temperature at $1.6 \mathrm{~m}$ for the VL's, and $1.3 \mathrm{~m}$ for the PF, during the selected Sols. 

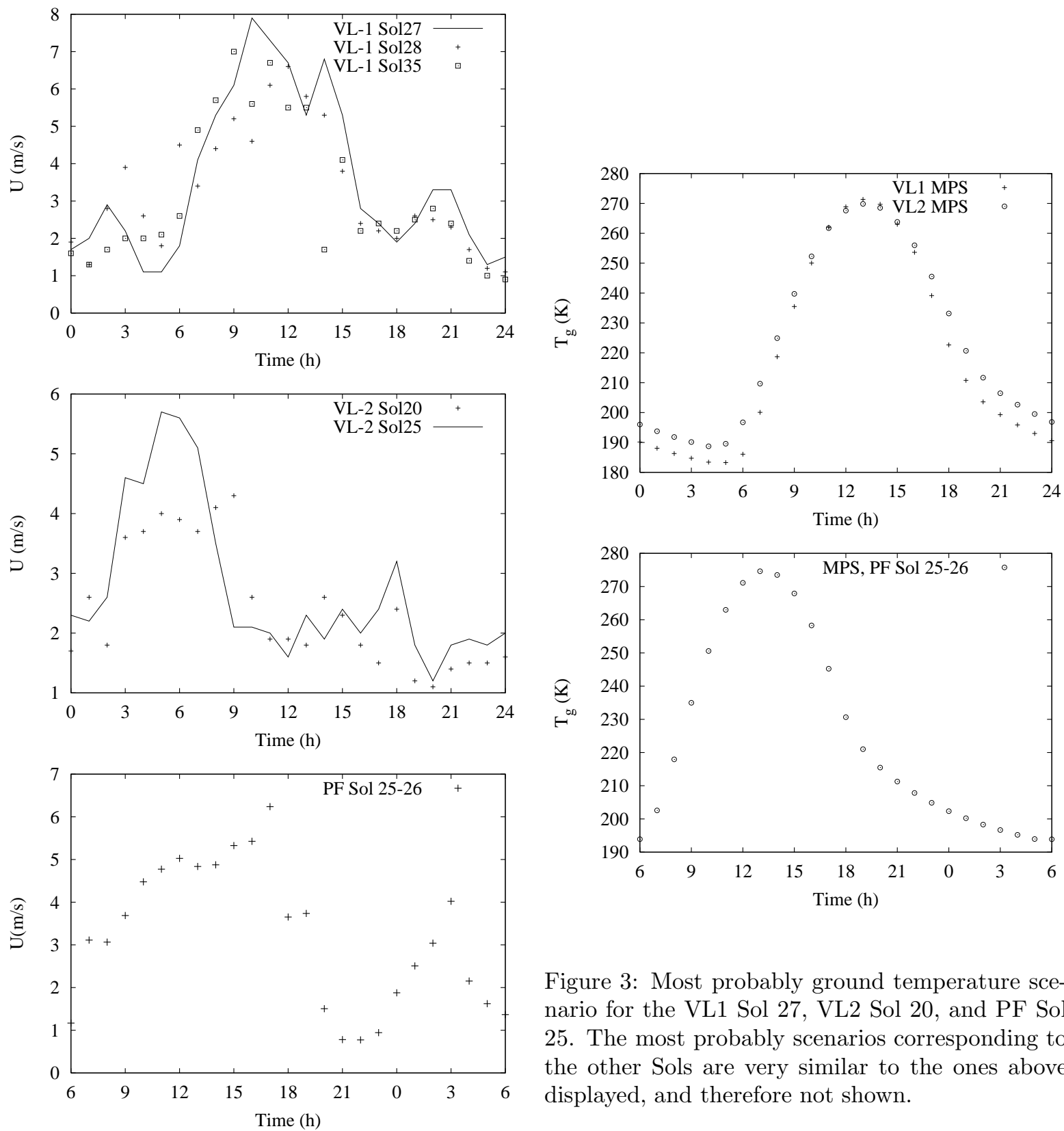

Figure 3: Most probably ground temperature scenario for the VL1 Sol 27, VL2 Sol 20, and PF Sol 25 . The most probably scenarios corresponding to the other Sols are very similar to the ones above displayed, and therefore not shown.

Figure 2: In situ horizontal wind speed at $1.6 \mathrm{~m}$ for the VL's, and $1.3 \mathrm{~m}$ for the PF, during the selected Sols. 
allows us to calculate the mixed layer height $z_{i}$, and hence, the convective velocity defined as $w_{*}=$ $\left(-u_{*} T_{*} z_{i} g / T_{g}\right)^{1 / 3}$, and also the convective temperature scale defined as $\theta_{*}=-u_{*} T_{*} / w_{*}$.

At this point we have values for the fundamental mixed layer similarity scales, namely: $z_{i}, w_{*}$, and $\theta_{*}$. Consequently, mixed layer similarity theory [7] could be applied in principle. However, its applicability on Mars is not as clear as on Earth. As we have employed this theory to obtain our results, we have created Appendix $\mathrm{C}$ to extensively explain the reliability of its use in the MML.

During the morning hours, in which convection is the dominant heating mechanism, we have calculated all the mixed layer magnitudes. Thus, $\left\langle\sigma_{w}\right\rangle$, $\left\langle\sigma_{u}\right\rangle$, and $\left\langle\sigma_{\theta}\right\rangle$ have been obtained by integrating along the MML the next mixed layer similarity relations

$$
\begin{gathered}
\frac{\sigma_{w}}{w_{*}}=(1.8)^{0.5}\left(1-0.8 z / z_{i}\right)^{1 / 3} \\
\frac{\sigma_{u}}{w_{*}} \simeq \frac{\left\langle\sigma_{u}\right\rangle}{w_{*}}=0.6 \\
\frac{\sigma_{\theta}}{\theta_{*}}=(1.8)^{0.5}\left(z / z_{i}\right)^{-1 / 3}
\end{gathered}
$$

with Eq. (3) from [13], Eq. (4) from [2], and Eq. (5) from 11. Finally, the mean turbulent viscous dissipation rate

$$
\langle\epsilon\rangle=\frac{0.5 w_{*}^{3}}{z_{i}}
$$

has been derived from 11. Notice that in this case, the term $2 \epsilon z_{i} / w_{*}^{3}$ formed by making $\epsilon$ nondimensional does not depend on $z / z_{i}$, as for $\sigma_{u}$ in (4). Thus, in both cases, the mean value across the mixed layer match the value at each height.

As an alternative procedure, we have calculated $\left\langle\sigma_{w}\right\rangle$ and $\left\langle\sigma_{\theta}\right\rangle$ from MSL parameters

$$
\begin{gathered}
\frac{\left\langle\sigma_{w}\right\rangle}{u_{*}}=0.8\left(z_{i} /|L|\right)^{1 / 3} \\
\frac{\left\langle\sigma_{\theta}\right\rangle}{T_{*}}=-1.2\left(z_{i} /|L|\right)^{-1 / 3}
\end{gathered}
$$

with Eqs. (17) and (8) from 33. The following conditions need to be met in order to obtain these two last relations: a balance between buoyancy and dissipation across the mixed layer, and a high correlation between temperature and vertical velocity turbulent fluctuations.

\section{Results}

The results are divided into three different subsections: (a) fundamental similarity scales, (b) turbulent statistics, and (c) turbulent viscous dissipation rate together with eddy transfer coefficients. In each of them, we distinguish between MML magnitudes and their MSL counterparts. Thus, concerning the fundamental similarity scales, $z_{i}, w_{*}$, and $\theta_{*}$ have been determined for the MML, and $L, u_{*}$, and $T_{*}$ for the MSL. With regards to the turbulent statistics, $\left\langle\sigma_{u}\right\rangle,\left\langle\sigma_{w}\right\rangle$, and $\left\langle\sigma_{\theta}\right\rangle$ have been determined for the MML, and also their MSL counterparts $\sigma_{u}^{z}, \sigma_{w}^{z}$, and $\sigma_{\theta}^{z}$ at the measuring height. Finally, $\langle\epsilon\rangle$ has been determined for the MML, and $\epsilon^{z}, k_{m}^{z}$, and $k_{h}^{z}$ for the MSL, also at the measuring height.

All the following figures and tables displayed in this section have been calculated under the most expectable scenario of the ground temperature (MPS, see Appendix B), after having included a parametrized molecular sublayer [4, and with the prescribed value of $z_{0}=1 \mathrm{~cm}$. That is, the most probably scenario. In addition, although not explicitly shown, we have also calculated all the magnitudes under the three simulated ground temperature scenarios, with and without the inclusion of the molecular sublayer, and for each of the given values of the surface roughness $z_{0}(0.1,1$, and 10 $\mathrm{cm})$. This has allowed us to perform sensitivity studies regarding the inclusion of the molecular sublayer (with fixed value of $z_{0}=1 \mathrm{~cm}$, and under the MPS), considering the surface roughness (under the MPS an with the molecular sublayer included), and considering the ground temperature scenario (with fixed value of $z_{0}=1 \mathrm{~cm}$ and with the inclusion of the molecular sublayer). These sensitivity studies will be shown in Section 5 .

\subsection{Fundamental similarity scales}

\subsubsection{Surface layer}

The approach followed to derive $L, u_{*}$, and $T_{*}$ can be seen in [16], where these magnitudes were calculated for the PF Sol 25. Instead of displaying their diurnal evolutions [something already done by other authors, as in 32 and 34], we show in table 2 our mean values obtained, noticing that by "mean values" we mean their average value during 
Table 2: Mean values of the fundamental surface layer similarity scales during the convective hours of the three missions. For the VL's, the values shown correspond to the average performed on a composite Sol formed by Sols 27, 28, and 35 for VL1, and Sols 20, and 25 for VL2.

\begin{tabular}{|l|l|l|l|}
\hline & VL-1 & VL-2 & PF \\
\hline$L(\mathrm{~m})$ & -40 & -7 & -25 \\
\hline$u_{*}\left(\mathrm{~m} \mathrm{~s}^{-1}\right)$ & 0.5 & 0.25 & 0.4 \\
\hline$T_{*}(\mathrm{~K})$ & -1.5 & -2.5 & -1 \\
\hline
\end{tabular}

the strongest convective hours.

For the Monin-Obukhov length, we have found it to lie between -7 and $-40 \mathrm{~m}$. Although not shown, it has been checked that Monin Obukhov length becomes slightly lower without the inclusion of the molecular sublayer, remaining the order of magnitude unchanged. It grows with the surface roughness, and, as it should be, $\left|L^{W}\right|<\left|L^{M P S}\right|<\left|L^{C}\right|$ under unstable situations. However, the absolute difference between $L^{M P S}$ and $L^{\text {extreme }}$ is never greater than 4 or $5 \mathrm{~m}$.

Concerning the friction velocity, we have obtained values between 0.25 and $0.50 \mathrm{~m} \mathrm{~s}^{-1}$, and have found that it shows virtually no difference whether the molecular sublayer is included or not. It has been also found that the friction velocity increases with the surface roughness, and, under unstable conditions, its value does not virtually change with the different ground temperature scenarios.

Finally, we have calculated scale temperature values to lie between -1 and $-2.5 \mathrm{~K}$. According to our results, it decreases, as we expected, about 35 $\%$ with the inclusion of the molecular sublayer. It does not show dependence on $z_{0}$, and the highest values are to be found for the warmest scenario $\left(\left|T_{*}^{W}\right|>\left|T_{*}^{M P S}\right|>\left|T_{*}^{C}\right|\right)$, since the difference in temperature between the ground level and one-meter-height is the maximum. Differences are quite small though, around $0.5 \mathrm{~K}$ between extreme values.

The dependence of the above magnitudes on the molecular sublayer is the expected one, because in this sublayer only molecular transfer is important for heat, whereas if we are considering momentum,

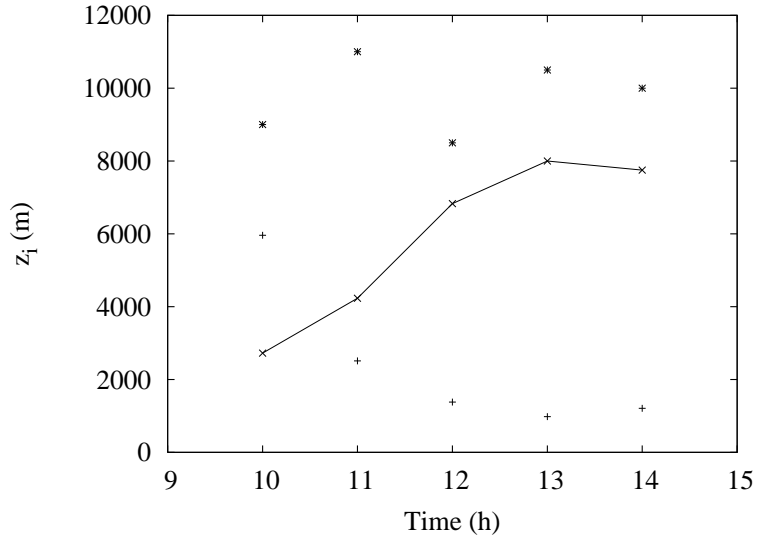

Figure 4: Mixed layer height during the strongest convective hours for the three missions. For the VL's, the values shown correspond to the average performed on a composite Sol formed by Sols 27, 28, and 35 for VL1, and Sols 20, and 25 for VL2. The solid line corresponds to the composite Sol of VL1 mission. VL2 composite Sol: +, and PF Sol: $*$.

pressure fluctuations - in addition to the molecular transport- are very relevant and therefore the net effect on the transport of momentum is less noticeable 38. Thus, the main consequence found with the inclusion of the molecular sublayer is a reduction of $T_{*}$ for a given difference between air temperature and surface skin temperature.

\subsubsection{Mixed Layer}

We have obtained the mixed layer height $z_{i}$ from Eq. (2). This relationship satisfies the fact that the ratio $\sigma_{u}^{z} / u_{*}$ becomes proportional to $w_{*}$ at large $-z_{i} / L$ values (convective conditions). Its evolution under strong convection for the three sites is displayed in Fig. (4). It can be noticed that $z_{i}$ values are around several kilometers height. These values match other $z_{i}$ estimations made using different approaches (1D models, LES's, or surface layer similarity theories). As an example, 9] 1D model calculates $z_{i} \simeq 4 \mathrm{~km}$ at noon for the VL1 summertime. 34 estimates $z_{i} \in(3.5,9.1) \mathrm{km}$ for both VL's sites. Concerning LES's, -for a generic run, 21 calculates $z_{i} \sim 7 \mathrm{~km}$, whereas [17] obtains $z_{i}^{\max } \simeq 6-7$ $\mathrm{km}$ for the PF site. Turning to the relations of 


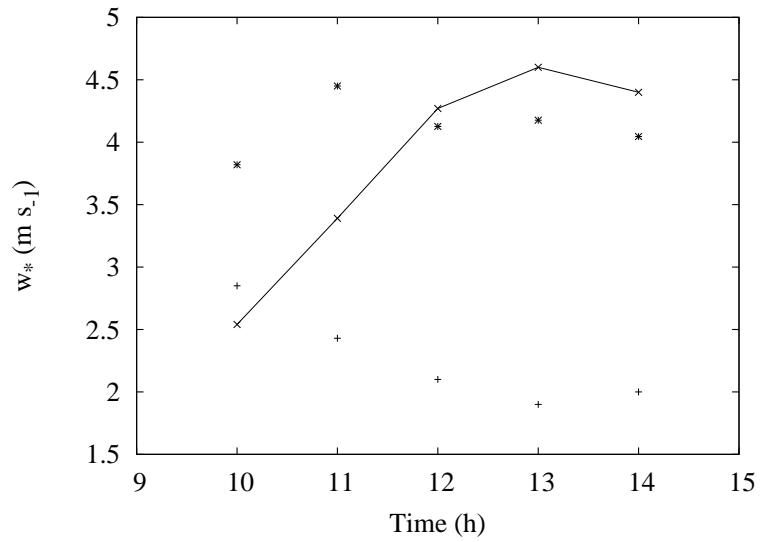

Figure 5: As in Fig. [4 but for the convective velocity scale.

dependence, it has been revealed that mixed layer height grows with the molecular sublayer, around 20-30\%, because, although $u_{*}$ is insensitive to its inclusion, $L$ does depend on it, see Eq. (2). Also from Eq. (2) is clear that $z_{i}$ decreases with $z_{0}$, since the $u_{*}$ surface roughness dependence becomes stronger than $L$ dependence, given that the former is raised to -3. Finally, as during the unstable hours $u_{*}$ remains unchanged under the three $T_{g}$ scenarios, and $\left|L^{C}\right|>\left|L^{M P S}\right|>\left|L^{W}\right|$, this all implies that $z_{i}^{C}>z_{i}^{M P S}>z_{i}^{W}$, the difference respect the MPS not being higher than $16 \%$.

The convective velocity scale has been obtained from its definition $w_{*}=\left(-u_{*} T_{*} z_{i} g / T_{g}\right)^{1 / 3}$, and its values are shown in Fig. 5 . They lie in the range 2 to $4.5 \mathrm{~m} \mathrm{~s}^{-1}$. Other Martian $w_{*}$ estimations, performed for a generic run, can be only found in [29] and [30, where values around $3.3 \mathrm{~m} \mathrm{~s}^{-1}$ are determined. Notice that the comparison with these two papers should be only performed near $10 \mathrm{am}$, because the Boussinesq approximation was employed there, and therefore, his results are restricted to shallow boundary layers, which would correspond to the early hours of the Martian day. This statement is valid for all the following comparisons made between our results and Sorbjan's ones. The convective velocity scale shows no dependence either on the inclusion of the molecular sublayer, nor on the surface roughness, nor on the different $T_{g}$ scenarios, as it could be expected from its definition and $u_{*}, T_{*}$, and $z_{i}$ relations of dependence.

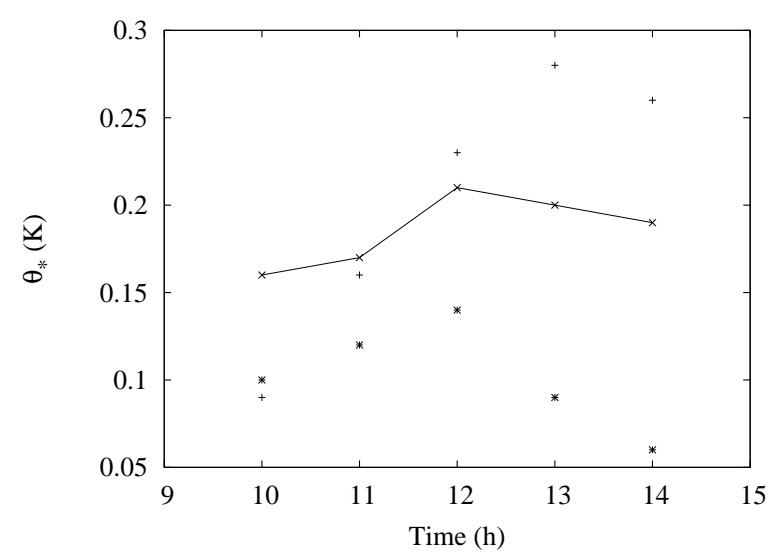

Figure 6: As in Fig. 4, but for the convective temperature scale.

The last fundamental mixed layer similarity scale that we have calculated is the convective temperature scale $\theta_{*}=-T_{*} u_{*} / w_{*}$. Its values for the three Martian sites are displayed in Fig. (6) and lie within the range 0.1 to $0.3 \mathrm{~K}$, similar to the $0.3 \mathrm{~K}$ value obtained in 29] for a generic run. It has been found that it decreases with the inclusion of the molecular sublayer via $T_{*}$, since $u_{*}$ and $w_{*}$ show no dependence. Although not physically expected, $\theta_{*}$ grows with the surface roughness a little bit, because $u_{*}$ dependence is not totally counteracted by $T_{*}$ dependence. Regarding $T_{g}$ sensitivity, it varies like $T_{*}$. That is, highest values for the $\mathrm{W}$ scenario, then for the MPS, and finally for the C. In any case, the variation between extremes values is smaller than $15 \%$.

\subsection{Turbulent Statistics}

\subsubsection{Surface layer}

Values of $\sigma_{u}^{z}$ and $\sigma_{T}^{z}$ have been straightforwardly inferred from the in situ temperature and velocity measurements at VL's and PF sites. Its mean values for the strongest convective hours can be seen in Table 3 As well, we have estimated $\sigma_{w}^{z}$ by using Eq. (11), and its results are shown in Fig. (17). $\sigma_{w}^{z}$ is around four times lower than $\sigma_{u}^{z}$, and so vertical turbulence is dominated by horizontal turbulence. This is mainly due to the fact that the measuring height was quite close to the ground. Standard 
Table 3: In situ mean magnitude of the temperature and horizontal wind speed standard deviations during the convective hours of the three missions at around $1.6 \mathrm{~m}$ (VL's) and $1.3 \mathrm{~m}(\mathrm{PF})$. For the VL's, the values shown correspond to the average performed on a composite Sol formed by Sols 27, 28, and 35 for VL1 site, and Sols 20, and 25 for VL2.

\begin{tabular}{|l|l|l|l|}
\hline & VL-1 & VL-2 & PF \\
\hline$\sigma_{u}^{z}\left(\mathrm{~m} \mathrm{~s}^{-1}\right)$ & 2.5 & 1.3 & 2.5 \\
\hline$\sigma_{T}^{z}(\mathrm{~K})$ & 3.0 & 2.7 & 3.5 \\
\hline
\end{tabular}

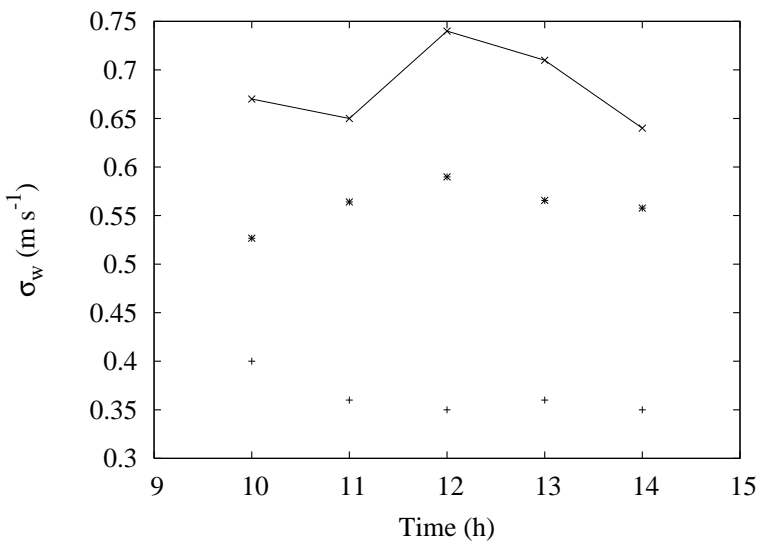

Figure 7: Vertical wind speed standard deviation during the strongest convective hours for the three missions. The solid line corresponds to the composite Sol of VL1 mission (at $1.6 \mathrm{~m}$ height). VL2 composite Sol: + (at $1.6 \mathrm{~m}$ height), and PF Sol: * (at $1.3 \mathrm{~m}$ height).

deviation values for vertical velocity are slightly higher without the inclusion of the molecular sublayer via $|L|$, although they are virtually negligible. It grows with the surface roughness $\left(u_{*}\right.$ dependence dominates $L$ dependence) and there is virtually no dependence on the ground temperature scenarios, although the higher values take place under the $\mathrm{W}$ scenario, because of the $L$ dependence.

\subsubsection{Mixed Layer}

We have derived the mean horizontal velocity standard deviation $\left\langle\sigma_{u}\right\rangle$ using Eq. (4). The mean value of this magnitude is expected to be representative

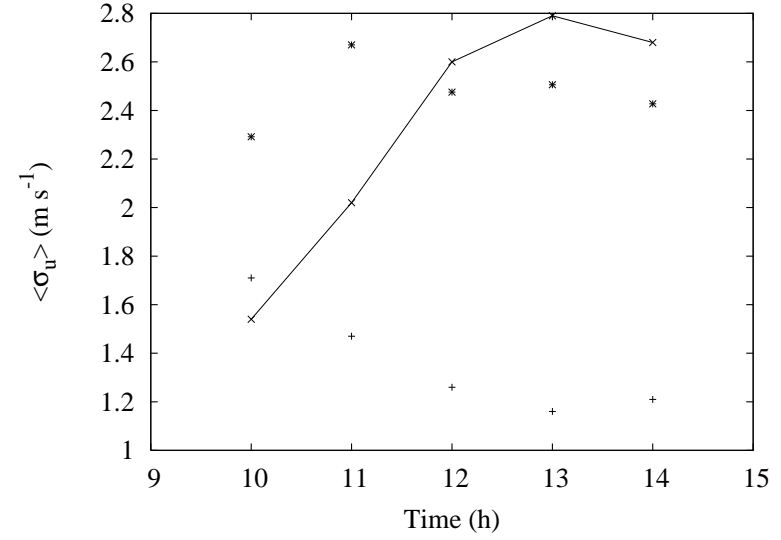

Figure 8: Mean mixed layer horizontal wind speed standard deviation during the strongest convective hours for the three missions. The solid line corresponds to the composite Sol of VL1 mission. VL2 composite Sol: + , and PF Sol: $*$.

of the whole MML, as $\sigma_{u} / w_{*}$ shows very little variation on $z / z_{i}$ in the Earth. $\left\langle\sigma_{u}\right\rangle$ values are shown in Fig. (8), and lie between 1.2 and $2.8 \mathrm{~m} \mathrm{~s}^{-1}$, which are slightly higher than those calculated by 29 for a generic run. It shows neither dependence on the molecular sublayer, nor on the surface roughness, nor on $T_{g}$ extreme scenarios, due to the $w_{*}$ relations of dependence.

To obtain $\left\langle\sigma_{w}\right\rangle$, we have employed two different approaches. On the one hand, mixed layer similarity has been used via Eq. (3), which, after having been integrated across the mixed layer, results in $\left\langle\sigma_{w}\right\rangle \simeq 0.55 w_{*}$. It is obvious that the use of the last relation demands that we question the reliability of the average value. That is, does $\sigma_{w}$ vary strongly across the mixed layer with regards to the mean value $\left\langle\sigma_{w}\right\rangle$ ? What we have obtained is that the order of magnitude of this variable primarily remains unchanged across the MML, whereby the reliability of this result becomes stronger. To prove it, $\sigma_{w}$ maximum values for all $z$ have been calculated using Eq. (3) with the extreme calculated values of $w_{*}$ and $z_{i}$. As a result we have always found the same order of magnitude. The second approach, Eq. (7), involves using the surface layer inputs. The results extracted from applying both techniques are shown in Fig. (9), where it can be seen that the values obtained are quite similar, and lie within the range $(1.2,2.8) \mathrm{m} \mathrm{s}^{-1}$. They also 

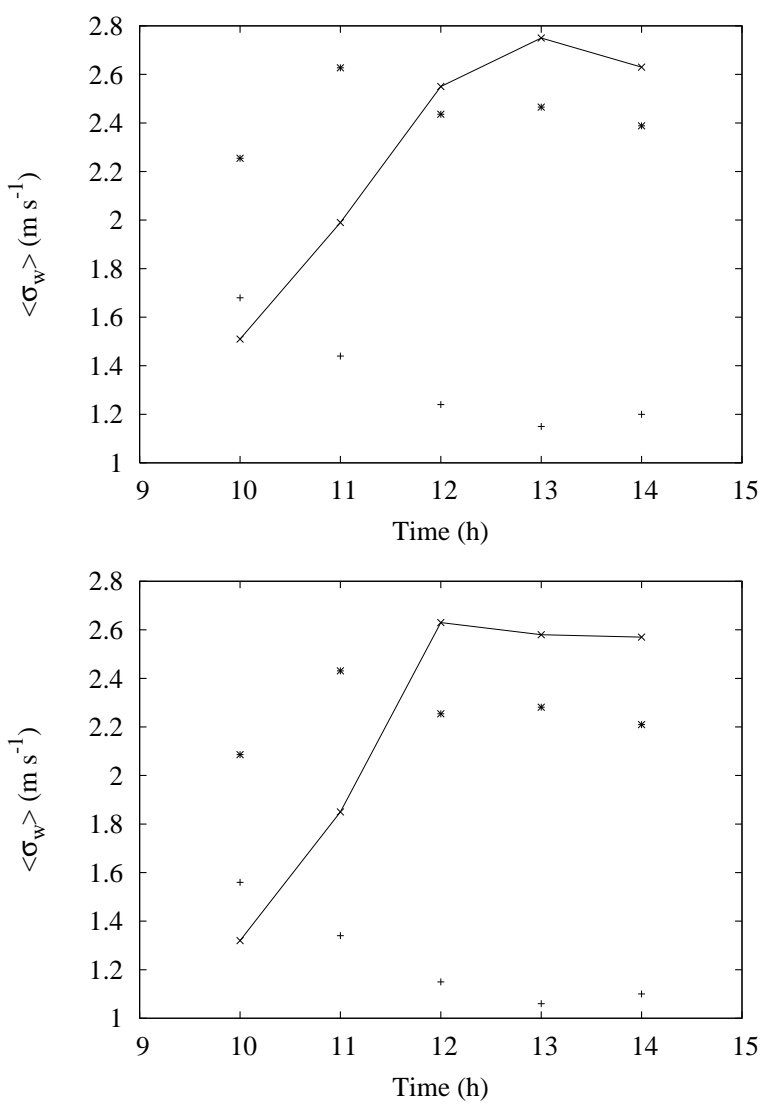

Figure 9: Mean mixed layer vertical wind speed standard deviation during the strongest convective hours for the three missions. Top figure corresponds to Eq. (7) and down figure to Eq. (3). In both cases, the solid line corresponds to the composite Sol of VL1 mission. VL2 composite Sol: +, and PF Sol: *. match those obtained in 21, and in 29, both using LES's for generic runs. Finally, the mean vertical wind speed standard deviation depends neither on the inclusion of the molecular sublayer, nor on the surface roughness, nor on the $T_{g}$ scenarios.

As for $\left\langle\sigma_{w}\right\rangle$, we have determined the mean temperature standard deviation $\left\langle\sigma_{\theta}\right\rangle$ by two procedures. The first involves mixed layer similarity, see Eq. (5), while surface layer parameters are involved in the second one, see Eq. (8). The mean temperature standard deviation is also expected to represent typical MML values, as extreme values for all $z$ of $\sigma_{\theta}$ do not exceed the order of magnitude of $\left\langle\sigma_{\theta}\right\rangle$. In Fig. [10] values of this parameter are displayed, mainly ranging from 0.2 to $0.5 \mathrm{~K}$. These values are similar to the ones shown in 29] for a generic run. Turning to the relations of dependence, $\left\langle\sigma_{\theta}\right\rangle$ values decrease with the inclusion of the molecular sublayer, given that $T_{*}$ also decreases and $u_{*}$, and $w_{*}$ remain unchanged. On the other hand, there virtually exists no variation regarding the surface roughness. Finally, maximum values are found under the $\mathrm{W}$ scenario, due to the $T_{*}$ dependence. However, just around $18 \%$ variation is found between the $\mathrm{W}$ and the $\mathrm{C}$ scenarios.

\subsection{Turbulent viscous dissipation rate, and eddy transfer coeffi- cients}

\subsubsection{Surface layer}

We have derived these magnitudes following the methodology explained in [16. Representative values for the three sites during the strongest convective hours can be seen in Table 4. Values corresponding to the eddy transfer coefficients are around $0.3-0.4 \mathrm{~m} \mathrm{~s}^{-2}$, the coefficient for heat transport being slightly higher. In the case of $\epsilon^{z}$, its maximum values are around $0.1 \mathrm{~m}^{2} \mathrm{~s}^{-3}$. Eddy transfer coefficients and turbulent viscous dissipation rate are slightly higher when calculated without molecular sublayer (around $6 \%$ for both magnitudes). They both grow with the surface roughness and remain almost unchanged under the three $T_{g}$ scenarios, although the $\mathrm{W}$ scenario shows slightly higher values for both magnitudes. 


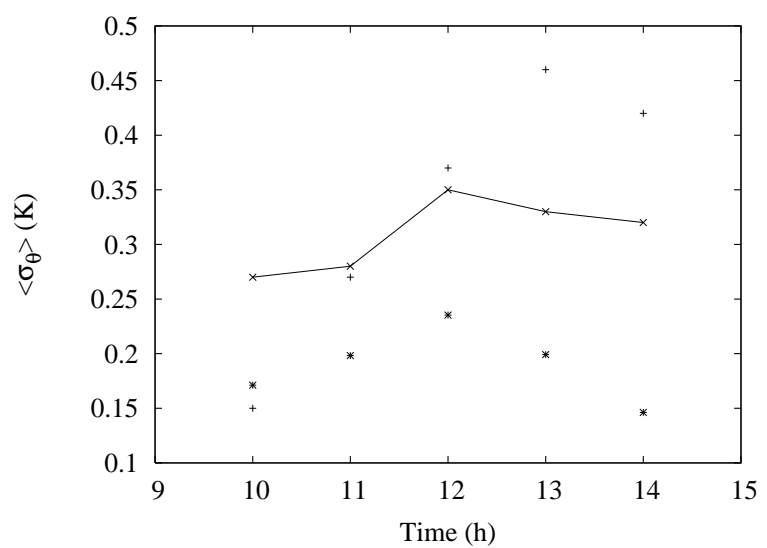

Table 4: Eddy diffusivity coefficients for momentum $k_{m}$ and heat $k_{h}$, and turbulent viscous dissipation rate $\epsilon$ during the convective hours for the three missions. These magnitudes have been calculated at around $1.6 \mathrm{~m}$ for VL's and $1.3 \mathrm{~m}$ for PF. The values shown correspond to the average performed on a composite Sol formed by Sols 27, 28, and 35 for VL1 site, and Sols 20, and 25 for VL2 site.

\begin{tabular}{|l|l|l|l|}
\hline & VL-1 & VL-2 & PF \\
\hline$k_{m}\left(\mathrm{~m} \mathrm{~s}^{-2}\right)$ & 0.4 & 0.2 & 0.25 \\
\hline$k_{h}\left(\mathrm{~m} \mathrm{~s}^{-2}\right)$ & 0.45 & 0.35 & 0.30 \\
\hline$\epsilon\left(\mathrm{m}^{2} \mathrm{~s}^{-3}\right)$ & 0.20 & 0.02 & 0.17 \\
\hline
\end{tabular}
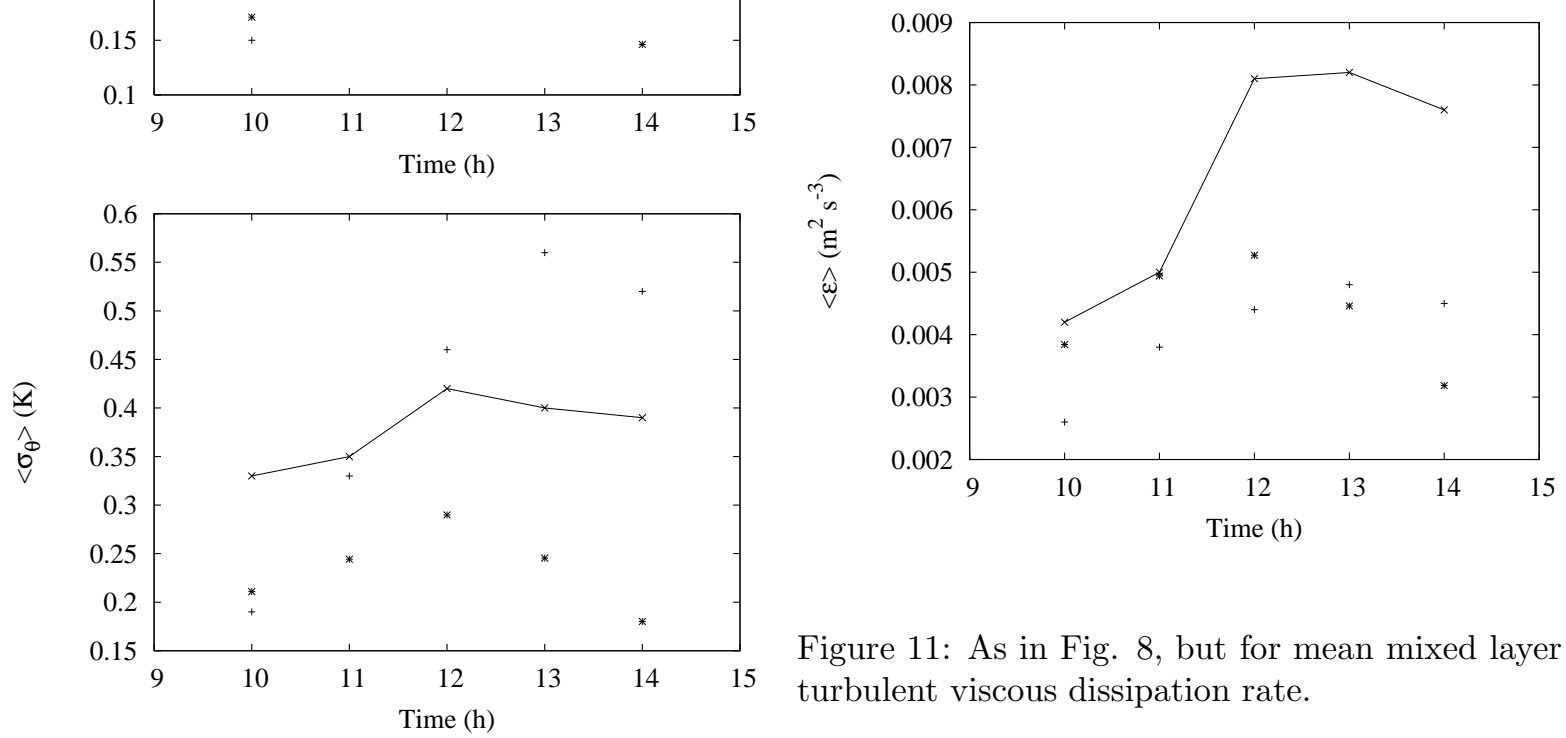

Figure 11: As in Fig. 8 but for mean mixed layer turbulent viscous dissipation rate.

Figure 10: Mean mixed layer temperature standard deviation during the strongest convective hours for the three missions. Top figure corresponds to Eq. (8) and down figure to Eq. (5). In both cases, the solid line corresponds to the composite Sol of VL1 mission. VL2 composite Sol: +, and PF Sol: *.

\subsubsection{Mixed Layer}

We have determined the mean mixed layer turbulent viscous dissipation rate $\langle\epsilon\rangle$ from Eq. (6). Its evolution is shown in Fig. 11] where it can be observed that its values are of the order of $0.005 \mathrm{~m}^{2}$ $\mathrm{s}^{-3}$. This is in accordance with the fact that dissipation balance the buoyancy term of the turbulent kinetic energy equation across the mixed layer, which has been supposed to derive Eq. (7) and Eq. (8). According to [33], $\left\langle\overline{w^{\prime} \theta^{\prime}}\right\rangle \simeq 0.2\left\langle\sigma_{w}\right\rangle\left\langle\sigma_{\theta}\right\rangle$ under strong convection. By substituting the values for $g=3.7 \mathrm{~m} \mathrm{~s}^{-2}, T_{g}=270 \mathrm{~K}$, and the mean values that we have obtained for the standard deviations, we obtain the value of order $0.005^{2} \mathrm{~s}^{-3}$ for the fac- 
tor $\left(g / T_{g}\right)\left\langle\overline{w^{\prime} \theta^{\prime}}\right\rangle$. That is, it matches the value we have estimated for $\langle\epsilon\rangle$. Regarding the relations of dependence, $\langle\epsilon\rangle$ decreases with the inclusion of the molecular sublayer since $z_{i}$ increases, see Eq. (6). Concerning the surface roughness, $\langle\epsilon\rangle$ grows with $z_{0}$, and finally, its values under the $\mathrm{W}$ scenario are around $20 \%$ higher than $\mathrm{C}$ scenario results.

\section{Discussion of the results}

\subsection{Comparison between MML and MLS magnitudes}

Focusing on the results, Tables [5] and 6] can not only be used to compare the MPBL magnitudes to their terrestrial counterparts, but also to compare the MSL magnitudes to their MML counterparts. Thus, we have found that turbulent temperature variations (standard deviations) become nearly as much ten times greater in the first meters of the MSL than in the averaged MML. The same can be said about the scale temperatures. This is something expected, since similarity theory has been used, and hence $\theta_{*} / T_{*} \propto\left(-z_{i} / L\right)^{-1 / 3}$ is satisfied. The converse is true for turbulent vertical wind speed variations, given that $w_{*} / u_{*} \propto\left(-z_{i} / L\right)^{1 / 3}$, and therefore $\left\langle\sigma_{w}\right\rangle$ is expected to be higher than $\sigma_{w}^{z}$. More concretely and according to our results, it is about five times higher. In the case of horizontal wind speed standard deviation, we have obtained similar values both for the MSL and for the MML. This in part due to the fact that even in the MSL, $\sigma_{u}^{z}$ scales with $w_{*}$ instead of with $u_{*}$, as it does in the MML.

\subsection{Sensitivity to surface roughness, to a molecular sublayer, and to ground temperature}

Taking into account that our results have been tested for different values of the surface roughness, under different ground temperature scenarios, and with and without the inclusion of a parametrized molecular sublayer, we summarize in Tables 7 and 8 their relation of dependence on these "external forcings".

Starting with temperature parameters $\left(T_{*}, \theta_{*}\right.$, and $\left.\left\langle\sigma_{\theta}\right\rangle\right)$, one may notice that they decrease if the inclusion of a molecular sublayer is carried out.
This is something to be expected,-- since, as it has been said before, the main consequence found upon the inclusion of the molecular sublayer, is the surface heat flux decrease for a given air temperature and surface skin temperature difference. However, its inclusion has no effect on wind parameters $\left(u_{*}\right.$, $\sigma_{w}^{z}, w_{*},\left\langle\sigma_{u}\right\rangle$, and $\left.\left\langle\sigma_{w}\right\rangle\right)$, because the net effect on the transport of momentum is less noticeable. The behavior of the calculated parameters under the different $T_{g}$ scenarios is the one that could be expected. That is, the higher the difference between the ground and the first meter (W scenario), the higher the value of these temperature parameters $\left(T_{*}, \theta_{*}\right.$, and $\left.\left\langle\sigma_{\theta}\right\rangle\right)$, whereas wind parameters show less sensitivity. Concerning the surface roughness, MSL parameters show stronger dependence on it, while MML parameters dependence is virtually negligible in almost all the cases.

\subsection{Comparison to the typical ter- restrial Planetary Boundary Layer}

Let us turn now to compare the values of the parameters obtained from the two planets. The Earth values shown correspond to planetary boundary layers formed over flat and homogeneous terrain, and under no baroclinic disturbances.

We start the comparison for the mixed layer on Mars and on Earth, see Table 6] The papers used to get the terrestrial results are: 31] for $z_{i}$; 13 for $w_{*}, \theta_{*}$, and $\left\langle\sigma_{w}\right\rangle$; 11 for $w_{*}, \theta_{*},\langle\epsilon\rangle$, and $\left\langle\sigma_{\theta}\right\rangle$, and, finally, [1] for $\left\langle\sigma_{u}\right\rangle$. Notice that almost all Martian values are higher than their terrestrial counterparts. Specifically, we have found that convective velocity scale and mean wind speed standard deviations are between two and four times higher on the MML, and hence, the mean turbulent kinetic energy. This was also pointed out in 21] using LES. That paper also mentioned that turbulence was not isotropic, as a result of vertical velocity variance being higher than the horizontal one. However, at least handling with mean values, similar values for both horizontal and vertical wind speed variances have been estimated in our calculations. Mean temperature standard deviation and convective temperature scale have also been found to be higher on Mars. This is something expectable, as $\left\langle\sigma_{\theta}\right\rangle$ is proportional to $\theta_{*}$, which in 
turn is also proportional to $T_{*}$, which is quite high because of the strong temperature gradients that prevail in the first Martian meters. Concerning the mixed layer height, as it has been already remarked by other authors, it is much higher on Mars, due to the much more vigorous existing convection.

We have carried out the same comparison with the characterizing surface layer parameters under unstable conditions, see Table [5] The articles consulted to get the terrestrial results are: $\left[5\right.$ for $\epsilon^{z}$, $\sigma_{\theta}^{z}$, and $\sigma_{w}^{z}$; 11] for $T_{*}$ and $\sigma_{w}^{z} ; 13$ for $T_{*}$, and finally [19] for $\sigma_{u}^{z}$. Remember that the Martian parameters have been estimated at $1.3 \mathrm{~m}$ for the $\mathrm{PF}$, and $1.6 \mathrm{~m}$ for the VL's, while for the terrestrial case, the height is indicated in Table 5 Typical values of $L, u_{*}, \sigma_{u}^{z}$, and $\sigma_{w}^{z}$ agree on both planets. On the other hand, $T_{*}$ and $\sigma_{\theta}^{z}$ are around one order of magnitude higher on Mars. The reason that lies behind these results can be explained as the sum of the following circumstances: (i) the net radiation that reaches the Martian soil is similar to the one reaching the terrestrial soil, (ii) sensible and latent fluxes are much lower on Mars (low atmospheric density and virtual absence of water vapor), (iii) the thermal inertia shows lower values on Mars, and (iv) the air atmospheric density is very low. This all implies much higher temperature gradients at the first meters and consequently higher values of $T_{*}$ and $\sigma_{\theta}^{z}$.

\section{Summary and Conclusions}

A complete research of the MPBL has been addressed in this paper. To do this, the main two layers that form the convective boundary layer have been characterized by determining several parameters. Values for the mixed layer height $z_{i}$, convective velocity scale $w_{*}$, convective temperature scale $T_{*}$, mean temperature standard deviation $\left\langle\sigma_{\theta}\right\rangle$, mean wind speed standard deviations $\left\langle\sigma_{u}\right\rangle$, and $\left\langle\sigma_{w}\right\rangle$, and mean turbulent viscous dissipation rate $\langle\epsilon\rangle$, all them calculated for the $\mathrm{PF}$ and VL's sites, have been derived in this paper concerning the MML. In addition, in order to characterize the MSL in this work, we have provided parameters which include, not only those already obtained such as the Monin-Obukhov length $L$, friction velocity $u_{*}$, and scale temperature $T_{*}$, but also new ones, such as vertical wind speed variance $\sigma_{w}^{z}$, tur- bulent viscous dissipation rate $\epsilon^{z}$, eddy transfer coefficients for heat $k_{h}^{z}$, and momentum $k_{m}^{z}$. Together with our values obtained, throughout the paper we can find references to other Martian articles in which some of the above mentioned magnitudes have also been derived by other methods. Moreover, numerical values of the all terrestrial counterparts are included, as well as their references.

Data-inputs employed to perform this study belong to the VL's and PF sites. Sols 27, 28, and 35 for the VL1, and Sols 20 and 25 for the VL2 have been chosen, while for the PF site it has only been selected Sol 25. Hourly in situ temperature and hourly in situ horizontal wind speed have been used from both missions, as well as simulated ground temperature. These all correspond to typical low mid latitudes northern summer time conditions, and thus the results of this work are representative of such conditions.

Concerning the methodology employed, MML parameters have been estimated by using mixed layer similarity theory. On the other hand, the methodology used to derive the MSL results is explained in [16]. Both for the MML and for the MSL, all and each of the magnitudes we have derived in this paper have been calculated under the different simulated ground temperature scenarios, with and without the inclusion of a parametrized molecular sublayer, and for the three proposed surface roughness values $(0.1,1$, and $10 \mathrm{~cm})$. By doing all this, we have constructed Tables 7 and 8 to show the relation of dependence between the results and the surface roughness, the molecular sublayer, and the ground temperature scenarios.

Let us now list the main conclusions of this work. Concerning the surface layer on both planets, we have derived similar values of $L, u_{*}, \sigma_{u}^{z}$, and $\sigma_{w}^{z}$, being an important factor for this result that on both planets horizontal wind speed in the first meters is quite similar. Yet scale temperature $T_{*}$ and temperature standard deviation $\sigma_{\theta}^{z}$ are around one order of magnitude higher on Mars. Regarding the mixed layer, $z_{i}$ values are much higher on Mars, mainly due to the much higher kinematic heat flux values prevailing on Mars. The mean standard deviation of horizontal $\left\langle\sigma_{u}\right\rangle$ and vertical $\left\langle\sigma_{w}\right\rangle$ wind speed are between two and four times higher on Mars, and hence the mean turbulent kinetic energy. Finally, the mean temperature standard deviation also becomes higher on Mars, given that it is pro- 
portional to $\theta_{*}$, and hence, also proportional to $T_{*}$, this last magnitude being around one order of magnitude higher on Mars.

It is worth mentioning the limitations of this work. As it has been explained in Appendix C, radiative heating, although non negligible, has been neglected. This means that our results are not generic for the whole Martian Planetary Boundary Layer, but for the low mid latitude northern summertime sites close to noon local times. That is, when convective heating dominates radiative heating the most. On the other hand, the set of similarity relationships used in this paper, although expected to be universal, have been calculated on Earth. Lastly, the ground temperature has not been measured in situ but is simulated, which constitutes another source of error. However, this kind of studies is a first and necessary step into a further understanding of the MPBL. In addition, we feel confident about the order of magnitude of the variables obtained in this work.

We believe that some actions should be carried out in next Martian missions, not only to make all these uncertainties vanish, but also to keep on improving our understanding of the MPBL (which remains an open question). In situ measurements of ground temperature (not measured yet) would be very useful, since this is an important parameter driving the evolution of the MPBL. Simultaneous measurements at different heights of temperature, humidity, and vertical (never measured) and horizontal wind speed would allow direct estimation of heat, humidity, and momentum surface fluxes. Moreover, Earth surface layer similarity relationships could be tested. In doing all this, the sampling rate should be high enough to capture small time scale processes associated to the microscale (typically higher than $1 \mathrm{~Hz}$ ). And the total sampling period should last no less than an hour. All these surface layer studies are within reach if we take consideration the current technology available to us. Direct measurements of MML magnitudes will probably take longer to happen.

\section{Acknowledgments}

We thank the MEC's projects CGL2005-06966C07-04, ESP2007-30839-E, and ESP2007-30487-E for their financial support. We also thank Prof.
Sävijärvi (University of Helsinki) for his advice in the use of his model, and Prof. Murphy (New Mexico University) for providing us wind data and helping us in the preparation of this paper. Prof. Luis Vázquez thanks the partial support from the Dirección General de Investigación of Spain, under grant MTM2005-05573. One of the authors thanks the fellowship from the firm Arquimea S.L., and also M. Feito and C. Cegarra for helping us in the realization of the paper. Finally, we thank the referees for their useful advises to improve this article. 
Table 5: Surface Layer turbulent parameters on Mars and on Earth. Terrestrial values correspond to planetary boundary layers formed over flat and homogeneous terrain, and under no baroclinic disturbances. Martian shown results have been calculated by us.

\begin{tabular}{|l|l|l|}
\hline Unstable Surface Layer & & \\
\hline & Mars & Earth \\
\hline$L$ & $-17 \mathrm{~m}$ & Similar \\
\hline$u_{*}$ & $\sim 0.4 \mathrm{~m} \mathrm{~s}^{-1}$ & $\sim 0.3 \mathrm{~m} \mathrm{~s}^{-1}$ \\
\hline$\left|T_{*}\right|$ & $\sim 1 \mathrm{~K}$ & $\sim 0.15-0.4 \mathrm{~K}$ \\
& & $\sim 0.27-0.88 \mathrm{~K}$ \\
\hline$\epsilon^{z}$ & $\sim 0.16 \mathrm{~m}^{2} \mathrm{~s}^{-3}$ & $\sim 0.01-0.02 \mathrm{~m}^{2} \mathrm{~s}^{-3}, z=4,32 \mathrm{~m}$ \\
& & $\sim 0.001-0.01 \mathrm{~m}^{2} \mathrm{~s}^{-3}, z=18 \mathrm{~m}$ \\
\hline$\sigma_{\theta}^{z}$ & $\sim 3 \mathrm{~K}$ & $\sim 0.18-0.32 \mathrm{~K}, z=4 \mathrm{~m}$ \\
\hline$\sigma_{u}^{z}$ & $\sim 2 \mathrm{~m} \mathrm{~s}^{-1}$ & $\sim 1.4 \mathrm{~m} \mathrm{~s}^{-1}, z=4 \mathrm{~m}$ \\
\hline$\sigma_{w}^{z}$ & $\sim 0.5 \mathrm{~m} \mathrm{~s}^{-1}$ & $\sim 0.4-0.6 \mathrm{~m} \mathrm{~s}^{-1}, z=4 \mathrm{~m}$ \\
& & $\sim 0.38-0.44 \mathrm{~m} \mathrm{~s}^{-1}, z=4,32 \mathrm{~m}$ \\
\hline
\end{tabular}


Table 6: As Table 5 but for the convective mixed layer.

\begin{tabular}{|l|l|l|}
\hline Convective Mixed Layer & & \\
\hline & Mars & Earth \\
\hline$z_{i}$ & $\sim 6 \mathrm{Km}$ & $\sim 0.2-2 \mathrm{Km}$ \\
\hline$w_{*}$ & $\sim 4 \mathrm{~m} \mathrm{~s}^{-1}$ & $\begin{array}{l}\sim 1.35-2.41 \mathrm{~m} \mathrm{~s}^{-1} \\
\sim 1-2 \mathrm{~m} \mathrm{~s}^{-1}\end{array}$ \\
\hline$\theta_{*}$ & $\sim 0.1 \mathrm{~K}$ & $\begin{array}{l}\sim 0.05-0.1 \mathrm{~K} \\
\end{array}$ \\
\hline$\langle\epsilon\rangle$ & & $\sim 0.03-0.07 \mathrm{~K}$ \\
\hline$\left\langle\sigma_{\theta}\right\rangle$ & $\sim 0.005 \mathrm{~m}^{2} \mathrm{~s}^{-3}$ & $\sim 0.001-0.005 \mathrm{~m}^{2} \mathrm{~s}^{-3}$ \\
\hline$\left\langle\sigma_{u}\right\rangle$ & $\sim 0.3 \mathrm{~K}$ & $\sim 0.06-0.2 \mathrm{~K}$ \\
\hline$\left\langle\sigma_{w}\right\rangle$ & $\sim 2.4 \mathrm{~m} \mathrm{~s}^{-1}$ & $\sim 0.47-1.13 \mathrm{~m} \mathrm{~s}^{-1}$ \\
\hline & $\sim 2.4 \mathrm{~m} \mathrm{~s}^{-1}$ & $\sim 0.6-1.4 \mathrm{~m} \mathrm{~s}^{-1}$ \\
\hline
\end{tabular}


Table 7: Dependencies on the molecular sublayer, on the surface roughness, and on the ground temperature scenarios for the calculated surface layer parameters under the strongest convective hours. $\nearrow$ means to grow with, while $\searrow$ to decrease with. The $\sim \nearrow$ and $\sim \searrow$ mean slightly increase with or slightly decrease with.

\begin{tabular}{|l|l|l|l|}
\hline & Molecular sublayer & Surface roughness & $T_{g}$ scenarios \\
\hline$|L|$ & $\sim \nearrow$ & $\nearrow$ & $\left|L_{C}\right|>\left|L_{M P S}\right|>\left|L_{W}\right|$ \\
\hline$u_{*}$ & $\mathrm{NO}$ & $\nearrow$ & $\mathrm{NO}$ \\
\hline$\left|T_{*}\right|$ & $\searrow$ & $\mathrm{NO}$ & $\left|T_{*}^{W}\right|>\left|T_{*}^{M P S}\right|>\left|T_{*}^{C}\right|$ \\
\hline$k_{h, m}^{z}$ & $\sim \searrow$ & $\nearrow$ & $k_{h, m}^{W} \geq k_{h, m}^{M S} \geq k_{h, m}^{C}$ \\
\hline$\epsilon^{z}$ & $\sim \searrow$ & $\epsilon^{W} \geq \epsilon^{M P S} \geq \epsilon^{C}$ \\
\hline$\sigma_{w}^{z}$ & $\sim \searrow$ & $\nearrow$ & $\sigma_{w}^{W} \geq \sigma_{w}^{M P S} \geq \sigma_{w}^{C}$ \\
\hline
\end{tabular}


Table 8: As Table $\mathbf{7}$ but for the calculated mixed layer parameters.

\begin{tabular}{|l|l|l|l|}
\hline & Molecular sublayer & Surface roughness & $T_{g}$ scenarios \\
\hline$z_{i}$ & $\nearrow$ & $\searrow$ & $z_{i}^{C}>z_{i}^{M P S}>z_{i}^{W}$ \\
\hline$w_{*}$ & $\mathrm{NO}$ & $\mathrm{NO}$ & $\mathrm{NO}$ \\
\hline$\theta_{*}$ & $\searrow$ & $\sim \nearrow$ & $\theta_{*}^{W}>\theta_{*}^{M P S}>\theta_{*}^{C}$ \\
\hline$\langle\epsilon\rangle$ & $\searrow$ & $\nearrow$ & $\langle\epsilon\rangle^{W}>\langle\epsilon\rangle^{M P S}>\langle\epsilon\rangle^{C}$ \\
\hline$\left\langle\sigma_{u}\right\rangle$ & $\mathrm{NO}$ & $\mathrm{NO}$ & $\mathrm{NO}$ \\
\hline$\left\langle\sigma_{w}\right\rangle$ & $\mathrm{NO}$ & $\mathrm{NO}$ & $\mathrm{NO}$ \\
\hline$\left\langle\sigma_{\theta}\right\rangle$ & $\searrow$ & $\sim \nearrow$ & $\left\langle\sigma_{\theta}\right\rangle^{W}>\left\langle\sigma_{\theta}\right\rangle^{M P S}>\left\langle\sigma_{\theta}\right\rangle^{C}$ \\
\hline
\end{tabular}




\section{Appendix A. List of Symbols and Definitions}

Monin Obukhov Length $L$. Scaling parameter used in the surface layer. Thought to represent a length above which buoyancy starts dominating shear.

Friction velocity $u_{*}$. Velocity scale in the surface layer. It is a measure of the surface drag.

Scale Temperature $T_{*}$. Temperature scale in the surface layer. Typical eddy temperature fluctuations in the surface layer.

Turbulent viscous dissipation rate $\epsilon^{z}$. It represents the conversion of turbulent kinetic energy into heat. Calculated at the measuring height.

Potential temperature variance $\sigma_{\theta}^{z}$. Calculated at the measuring height and averaged in the interval of 1 hour.

Horizontal wind speed variance $\sigma_{u}^{z}$. Calculated at the measuring height and averaged in the interval of 1 hour.

Vertical wind speed variance $\sigma_{w}^{z}$. Simulated at the measuring height.

Mixed layer height $z_{i}$. Often defined as the most negative heat flux level.

Convective scaling velocity $w_{*}$. It is a scaling velocity for the mixed layer. Its value match the magnitude of the vertical velocity fluctuations in thermals.

Convective temperature scale $\theta_{*}$. It is a scaling temperature for the mixed layer. Its values approximately represent how much warmer thermals are than the environment.

Mean mixed layer turbulent viscous dissipation rate $\langle\epsilon\rangle$. Conversion of turbulent kinetic energy into heat. Averaged over the whole mixed layer.

Mean mixed layer potential temperature variance $\left\langle\sigma_{\theta}\right\rangle$. It corresponds to the average over the whole mixed layer.

Mean mixed layer horizontal wind speed variance $\left\langle\sigma_{u}\right\rangle$. It corresponds to the average over the whole mixed layer.

Mean mixed layer vertical wind speed variance $\left\langle\sigma_{w}\right\rangle$. It corresponds to the average over the whole mixed layer. 


\section{Appendix B. Simulated Ground Temperature}

We have used a version of the 1D model presented in [25] to simulate the ground temperature during the selected Sols at the three different sites. As the model needs some external parameters to be run, we have taken advantage of articles in which those external parameters that influence simulated $T_{g}$ the most (thermal inertia, albedo, dust optical depth, surface emissivity,...) have been estimated, as in [6] and 20], or have simply been imposed, as in existing articles involving the Sävijärvi 1D model and 9 . Within the found range of values of these external parameters, we have simulated three $T_{g}$ scenarios: the most probably scenario (MPS), and the extreme warmest (W) and coldest (C) scenarios (expected and reliable extreme scenarios of ground temperature). For the MPS it has been imposed that the match between in situ and modeled air temperature, at $1.3 \mathrm{~m}$ for the $\mathrm{PF}$ and $1.6 \mathrm{~m}$ for the VK's, must be the best. As a result, the external parameters used to run the model for the MPS can be seen in Table 9 In creating the extreme scenar-

Table 9: 1D Model parameters used to create the MPS for VL-1 Sols 27, 28, 35, VL-2 Sols 20, 25, and $\mathrm{PF}$ Sol 25. $T(1)$ is the initial ground temperature, $G A$ refers to the initial vertical temperature lapse rate, $U G 0$ is the geostrophic wind speed, $z_{0}$ is the surface roughness, $G i$ is the thermal inertia, $\tau$ is the dust optical depth, $\alpha$ the surface albedo, and $S E$ the surface emissivity. and remains unaltered at night-time when albedo is decreased.

The superscript $W, C$, and MPS will be added throughout the paper to any magnitude (for instance, $L^{W}, L^{M P S}$, and $L^{C}$ ) to denote that they have been calculated under the warmest, coldest, and most probably scenario accordingly.

\begin{tabular}{|l|l|l|l|l|l|l|l|l|}
\hline & $T(1)(\mathrm{K})$ & $G A(\mathrm{~K} / \mathrm{Km})$ & $U G 0\left(\mathrm{~m} \mathrm{~s}^{-1}\right)$ & $z_{0}(\mathrm{~m})$ & $G i(\mathrm{~S} . \mathrm{I})$ & $\tau$ & $\alpha$ & $S E$ \\
\hline VL-1 & 215 & 2 & 10 & 0.01 & 269 & 0.4 & 0.22 & 0.95 \\
\hline VL-2 & 215 & 2 & 0 & 0.01 & 234 & 0.5 & 0.23 & 0.90 \\
\hline PF & 225 & 2 & 10 & 0.01 & 387 & 0.2 & 0.19 & 0.90 \\
\hline
\end{tabular}

ios, extreme values found of these parameters have been used to run the model, having in mind that: $T_{g}$ increases when surface emissivity decreases, $T_{g}$ increases during daytime and decreases at nighttime when thermal inertia or dust optical depth are decreased, and finally $T_{g}$ increases during daytime 


\section{Appendix C. On the applicability of the mixed layer similarity theory on Mars}

The main hypotheses which must be satisfied when applying mixed layer similarity theory are: (i) convection is the dominant heating mechanism throughout the mixed layer, and (ii) surface stress effects become negligible through this layer. This is certainly so in the Earth under fair weather conditions [1, because the heating rate due to the radiation divergence is negligible, and the winds are light under the mentioned conditions. On Mars, special care must be taken though. There is no uncertainty in neglecting the effects of the surface stress through the bulk of the MML, since the winds were calm during the Sols under study (low mid latitude northern summertime). The problem arises with the radiative heating throughout the MML, which becomes now non negligible due to the long wave radiative heating $\left(\mathrm{CO}_{2}\right.$ atmosphere), and also to the absorption of solar radiation by dust.

Two questions emerge immediately: how important is the radiative heating compared to the convective heating? And consequently, to what extent are our results reliable after having been calculated using a theory which neglects this radiative heating? Based on our results performed by a modified version of [25], and supported by [22], 9], and [24], the convective heating is between two and three times higher than the radiative one through the bulk of the MML, specially close to noon. In 29, this ratio can even reach a factor of five for early morning hours. The thin northern summertime atmosphere and the low atmospheric dust load existing during the Sols under study reduce the radiative heating strength, favoring the convective heating domain. Thus, convection still becomes the dominant mechanism, although radiative divergence can not be ignored. All this leads us to keep using mixed layer similarity, while remembering the above discussion and the corresponding limitations (time of the year and time of the day). Notice also that the results shown in the displayed figures range from $10 \mathrm{am}$ to $14 \mathrm{am}$. Based on our results, convective heating becomes higher than the radiative one during these hours, and specially near noon, where the first becomes around three times higher.

Once the first question has been answered, we move on to the second one. 29] found that magnitudes such as temperature and wind speed MML standard deviation do not change their order of magnitude after having introduced weak and strong radiative heating (see figures 7 and 10 of that work). With regards to the reliability of our results, we also expect them to be reliable concerning the order of magnitude, given that we have performed our research in the most favorable time of the year and of the day, to avoid the issue of radiative heating. 


\section{References}

[1] J. C. André, G. De Moor, P. Lacarreré, G. Therry, and R. du Vachar. Modeling the 24-hour evolution of the mean and turbulent structure of the planetary boundary layer. $J$. Atmos. Sci., 35:1861-1883, 1978.

[2] P.S. Arya. Introduction to Micrometeorology. Academic Press, 2001.

[3] S.L. Blumsack, P.J. Gierasch, and W.R. Wessel. An Analytical and Numerical Study of the Martian Planetary Boundary Layer Over Slopes. Journal of the Atmospheric Sciences, 30(1):66-82, 1973.

[4] HW Brutsaert. Exchange processes at the earth-atmosphere interface. Engineering $\mathrm{Me}$ teorology (Plate E, ed.). Elsevier: New York, pages 319-369, 1982.

[5] FH Champagne, CA Friehe, JC LaRue, and JC Wynagaard. Flux Measurements, Flux Estimation Techniques, and Fine-Scale Turbulence Measurements in the Unstable Surface Layer Over Land. Journal of the Atmospheric Sciences, 34(3):515-530, 1977.

[6] PR Christensen, JL Bandfield, VE Hamilton, SW Ruff, GL Mehall, N. Gorelick, K. Bender, K. Murray, HH Kieffer, and TN Titus. Mars Global Surveyor Thermal Emission Spectrometer experiment- Investigation description and surface science results. Journal of Geophysical Research, 106(E10):23823-23872, 2001.

[7] J.W. Deardorff. Convective Velocity and Temperature Scales for the Unstable Planetary Boundary Layer and for Rayleigh Convection. Journal of the Atmospheric Sciences, 27(8):1211-1213, 1970.

[8] P. Gierasch and R. Goody. A study of the thermal and dynamical structure of the martian lower atmosphere. Planetary and Space Science, 16(5):615-646, 1968.

[9] R.M. Haberle, H.C. Houben, R. Hertenstein, and T. Herdtle. A Boundary-Layer Model for Mars: Comparison with Viking Lander and Entry Data. Journal of the Atmospheric Sciences, 50(11):1544-1559, 1993.
[10] SL Hess, RM Henry, CB Leovy, JE Tillman, and JA Ryan. Meteorological results from the surface of Mars-Viking 1 and 2. Journal of Geophysical Research, 82:4559-4574, 1977.

[11] J. C. Kaimal, J. C. Wyngaard, D. A Haugen, O. R. Cote, and Y. Izumi. Turbulence Structure in the Convective Boundary Layer. Journal of the Atmospheric Sciences, 33:21522161, 1976.

[12] SE Larsen, HE Jørgensen, L. Landberg, and JE Tillman. Aspects Of The Atmospheric Surface Layers On Mars And Earth. BoundaryLayer Meteorology, 105(3):451-470, 2002.

[13] DH Lenschow, JC Wyngaard, and WT Pennell. Mean-Field and Second-Moment Budgets in a Baroclinic, Convective Boundary Layer. Journal of the Atmospheric Sciences, 37(6):1313-1326, 1980.

[14] A. Määttänen and H. Savijärvi. Sensitivity Tests with a One-Dimensional BoundaryLayer Mars Model. Boundary-Layer Meteorology, 113(3):305-320, 2004.

[15] SJ. Magalhaes and P. Gierasch. A Model of the Martian Slope Winds: Implications for Aeolian Transport. Journal of Geophysical Research, 87:9975-9984, 1982.

[16] G.M. Martinez, F. Valero, and L. Vazquez. Characterization of the Martian Surface Layer. Journal of the Atmospheric Sciences, Accepted, 2008.

[17] T.I. Michaels and S.C.R. Rafkin. Large-eddy simulation of atmospheric convection on Mars. Quarterly Journal of the Royal Meteorological Society, 130(599):1251-1274, 2002.

[18] M. Odaka. A numerical simulation of Martian atmospheric convection with a twodimensional anelastic model: A case of dustfree Mars. Geophys. Res. Lett, 28(5):895-898, 2001.

[19] H.A. Panofsky, H. Tennekes, D.H. Lenschow, and J.C. Wyngaard. The characteristics of turbulent velocity components in the surface layer under convective conditions. Boundary-Layer Meteorology, 11(3):355-361, 1977. 
[20] N.E. Putzig, M.T. Mellon, K.A. Kretke, and R.E. Arvidson. Global thermal inertia and surface properties of Mars from the MGS mapping mission. Icarus, 173(2):325-341, 2005.

[21] S.C.R. Rafkin, R.M. Haberle, and T.I. Michaels. The Mars Regional Atmospheric Modeling System: Model Description and Selected Simulations. Icarus, 151(2):228-256, 2001.

[22] H. Savijärvi. A model study of the PBL structure on Mars and the earth. Beitraege zur Physik der Atmosphaere, 64:219-229, 1991.

[23] H. Savijärvi. Mars Boundary Layer Modeling: Diurnal Moisture Cycle and Soil Properties at the Viking Lander 1 Site. Icarus, 117(1):120127, 1995.

[24] H. Savijärvi. A model study of the atmospheric boundary layer in the Mars Pathfinder lander conditions. Quarterly Journal of the Royal Meteorological Society, 125(554):483493, 1999.

[25] H. Savijärvi, A. Määttänen, J. Kauhanen, and A.M. Harri. Mars Pathfinder: New data and new model simulations. Quarterly Journal of the Royal Meteorological Society, 130(597):669-683, 2004.

[26] H. Savijärvi and T. Siili. The Martian Slope Winds and the Nocturnal PBL Jet. Journal of the Atmospheric Sciences, 50(1):77-88, 1993.

[27] JT Schofield, JR Barnes, D. Crisp, RM Haberle, S. Larsen, JA Magalhaes, JR Murphy, A. Seiff, and G. Wilson. The Mars Pathfinder Atmospheric Structure Investigation/Meteorology (ASI/MET) Experiment. Science, 278(5344):1752, 1997.

[28] T. Siili, RM Haberle, and JR Murphy. Sensitivity of Martian Southern polar cap edge winds and surface stresses to dust optical thickness and to the large-scale sublimation flow. Advances in Space Research, 19(8):12411244, 1997.

[29] Z. Sorbjan. Statistics of shallow convection on Mars based on large-eddy simulations. Part 1: shearless conditions. Boundary-Layer Meteorology, 123(1):121-142, 2007.
[30] Z. Sorbjan. Statistics of shallow convection on Mars based on large-eddy simulations. Part 2: effects of wind shear. Boundary-Layer Meteorology, 123:143-157, 2007.

[31] R.B. Stull. An Introduction to Boundary Layer Meteorology. Springer, 1988.

[32] J.L. Sutton, C.B. Leovy, and J.E. Tillman. Diurnal Variations of the Martian Surface Layer Meteorological Parameters During the First 45 Sols at Two Viking Lander Sites. Journal of the Atmospheric Sciences, 35(12):2346-2355, 1978.

[33] H. Tennekes. Free Convection in the Turbulent Ekman Layer of the Atmosphere. Journal of the Atmospheric Sciences, 27(7):1027-1034, 1970.

[34] J.E. Tillman, L. Landberg, and S.E. Larsen. The Boundary Layer of Mars: Fluxes, Stability, Turbulent Spectra, and Growth of the Mixed Layer. Journal of the Atmospheric Sciences, 51(12):1709-1727, 1994.

[35] A.D. Toigo and M.I. Richardson. A mesoscale model for the Martian atmosphere. J. Geophys. Res, 107(E7):3-1, 2002.

[36] A.D. Toigo, M.I. Richardson, S.P. Ewald, and P.J. Gierasch. Numerical simulation of Martian dust devils. J. Geophys. Res, 108(6):1-14, 2003.

[37] ZJ Ye, M. Segal, and RA Pielke. A Comparative Study of Daytime Thermally Induced Upslope Flow on Mars and Earth. Journal of the Atmospheric Sciences, 47(5):612-628, 1990.

[38] X. Zeng and R.E. Dickinson. Effect of Surface Sublayer on Surface Skin Temperature and Fluxes. Journal of Climate, 11(4):537$550,1998$. 